\title{
Reconfiguration of Strategy for Hand Surgery during COVID-19 Pandemic: Our Experience
}

\author{
Ankit Khurana ${ }^{1}$, Shailendra C Gupta ${ }^{2}$, Kuldeep Malik ${ }^{3}$, Vishal Jain ${ }^{4}$, Ishoo R Taneja ${ }^{5}$
}

\begin{abstract}
The present correspondence presents the author's experience with adherence to the wide awake local anesthesia no tourniquet (WALANT) technique for hand surgery as has been recommended in the pandemic. The authors plan to hold on to the use of daycare hand surgery under WALANT in our practice as a long-lasting modification as we emerge in the post-COVID following the vaccination drive.

Keywords: COVID-19, Hand surgery, Pandemic, Wide awake local anesthesia no tourniquet, Wide awake hand surgery.

Journal of Orthopedics and Joint Surgery (2021): 10.5005/.jp-journals-10079-1043
\end{abstract}

Level of evidence: $\mathrm{V}$

The Indian Orthopaedic Association has issued guidelines stating that orthopedic surgery should be reserved for emergency and obligatory situations, with the use of universal infection control precautions and avoiding unnecessary hospital visits and encouraging the use of telemedicine as far as possible. ${ }^{1}$ The British Society for Surgery of the Hand (BSSH), British Orthopaedic Association (BOA), and Orthopaedic Trauma Society (OTS) have recommended wide awake local anesthesia no tourniquet (WALANT) for hand and wrist surgery during the COVID-19 pandemic. ${ }^{2-4}$ The recommendation for WALANT arose due to the crisis in theaters and limited anesthetic support during the pandemic. This allowed for work flexibility and better utilization of existing limited resources. Wide awake local anesthesia no tourniquet provides a cheap, efficient, and safe method of hand surgery in resource-constrained scenarios. ${ }^{5}$ This technique also allows for active motion to be preserved so that results of surgery can be confirmed intraoperatively and inadvertent structural damage can be managed immediately.

Beyond this technique's clinical benefits, there is avoidance of the cost associated with preoperative anesthesia clearance, surgical

\footnotetext{
${ }^{1-4}$ Department of Orthopaedics, ESI Hospital, Rohini, Delhi, India ${ }^{5}$ Department of Anesthesia, ESI Hospital, Rohini, Delhi, India
}

Corresponding Author: Ankit Khurana, Department of Orthopaedics, ESI Hospital, Rohini, Delhi, India, Phone: +91 8437029392, e-mail: ankit24388@gmail.com

How to cite this article: Khurana A, Gupta SC, Malik K, et al. Reconfiguration of Strategy for Hand Surgery during COVID-19 Pandemic: Our Experience. J Orth Joint Surg 2021;3(1):63-64.

Source of support: Nil

Conflict of interest: None

supply costs, and avoidance of the costs associated with equipment and staff required for operating rooms. ${ }^{6}$ Moreover, patient's stay in the healthcare facility is reduced and the overall financial burden to the already strained healthcare system is decreased. This led to a paradigm shift in the management of hand and wrist surgery cases which were done in the hand unit of the Department of Orthopaedics and our services adopted rapidly and expanded the use of WALANT (Figs 1 and 2).
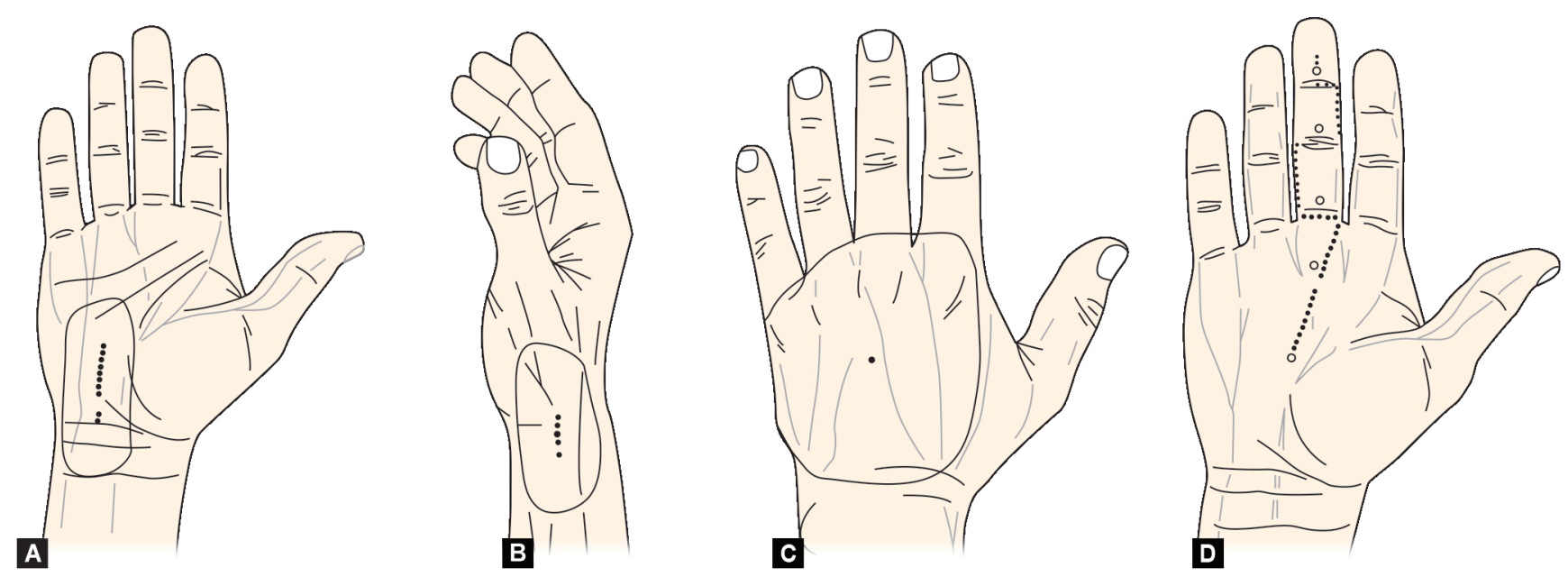

Figs 1 A to D: Injection site for $1 \%$ lignocaine with 1 in 100,000 epinephrine (buffered with $10 \mathrm{~mL}$ lido/epi: $1 \mathrm{~mL}$ of $8.4 \%$ sodium bicarbonate) under the skin along the dotted line for (A) Carpal tunnel release; (B) Dequervain's tenosynovitis release; (C) Ganglion excision; (D) Tendon exploration/repair

(c) The Author(s). 2021 Open Access This article is distributed under the terms of the Creative Commons Attribution 4.0 International License (https:// creativecommons.org/licenses/by-nc/4.0/), which permits unrestricted use, distribution, and non-commercial reproduction in any medium, provided you give appropriate credit to the original author(s) and the source, provide a link to the Creative Commons license, and indicate if changes were made. The Creative Commons Public Domain Dedication waiver (http://creativecommons.org/publicdomain/zero/1.0/) applies to the data made available in this article, unless otherwise stated. 


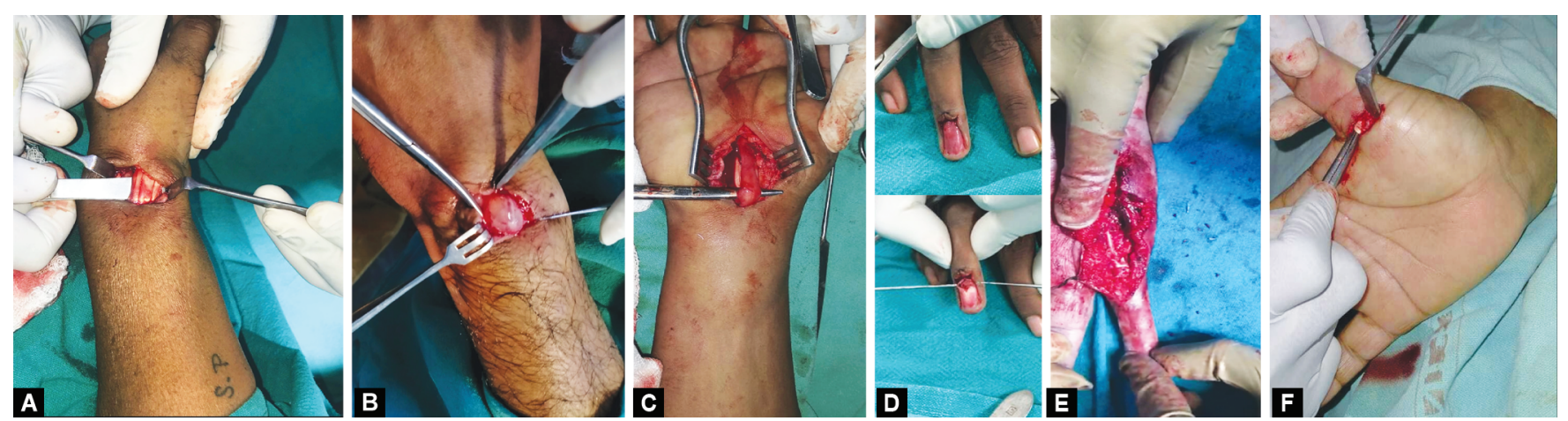

Figs 2A to F: Wide awake hand surgeries carried out during the pandemic (A) Dequervain's tenosynovitis, (B) Ganglion excision; (C) Carpal tunnel release; (D) Glomus tumor excision; (E) Tendon and $A 2$ pulley repair of the 5 th digit; $(F)$ Trigger thumb release

As the patients requiring hand surgery presented to our hospital during the peak of the pandemic, we needed to provide a system that treated patients smoothly while simultaneously preventing COVID-19 intra-hospital transmission at all costs. The importance of this was highlighted in a January 2020 case from Wuhan, where a hand surgeon contracted COVID-19 from a symptomatic patient during an emergency hand surgery. ${ }^{7}$ Owing to this case and further reports of COVID-19 infection in OT scenarios, a survey was carried out from 47 hand surgeons from 34 countries which found that the protocols for hand surgery units varied in terms of visitors, occupational health professionals, patient waiting areas, wards, and emergency rooms. Based on these preliminary findings, a rapid international consensus on hand surgery practices is needed for the current and future viral pandemic. ${ }^{8}$

Picardo et al. have published results of WALANT from Birmingham Hand Center during the present pandemic which underwent a dramatic service reconfiguration allowing treatment of patients requiring hand surgery to endure the pandemic height. The authors found that there was a substantial decrease in patient footfall and hospital admission, thereby eliminating COVID-19 transmission intra-hospital. ${ }^{9}$ Similar service reconfiguration has been suggested by Venkatesan et al. as well who have suggested the use of Mini $C$ arm and WALANT in hand surgery cases. ${ }^{10}$

Our experience of adherence to the WALANT technique for hand surgery has been recommended in the pandemic. In the future, the authors recommend applying this technique to more soft tissue procedures such as tendon repair and transfers, phalangeal fracture fixation, and small joint fusions in our hospital and suggest using this technique more widely. The medical fraternity has the responsibility of providing a continuity of care for patients has to be balanced with measures to reduce the risk of viral transmission. Wide awake local anesthesia no tourniquet allowed us to apply the COVID-19 BSSH guidelines in the management of surgical conditions of the hand and wrist in the current pandemic, thus providing adequate hand surgery support while simultaneously allowing safety not only to the surgeons but also to the patient.

The paradigm shift with the use of WALANT allowed the authors to streamline hand surgery services by substantially decreasing chances of COVID-19 transmission, decreased inpatient admission, and simultaneously resulted in high patient satisfaction. The authors plan to hold on to the use of daycare hand surgery under WALANT in our practice as a long-lasting modification as we emerge in the post-COVID age with the current vaccination drive. This will allow us to maintain a stable service should a future pandemic occur or the strain mutates.

\section{References}

1. Indian Orthopaedic Assocation. https://www.ioaindia.org/.

2. Hobday $D$, Welman $T, O^{\prime} N$ eill $N$, et al. A protocol for wide awake local anaesthetic no tourniquet (WALANT) hand surgery in the context of the coronavirus disease 2019 (COVID-19) pandemic. The Surgeon 2020;18(6):e67-e71. DOI: 10.1016/j.surge.2020. 06.015 .

3. BSSH, COVID-19 resources for members. https://www.bssh.ac.uk/ about/news/163/covid19_resources_for_members.

4. Management of patients with urgent orthopaedic conditions and trauma during the coronavirus pandemic. https://www.boa.ac.uk/ uploads/assets/ee39d8a8-9457-4533-9774e973c835246d/4e3170c2d85f-4162-a32500f54b1e3b1f/COVID-19-BOASTs-Combined-FINAL. pdf.

5. Lalonde DH. Latest advances in wide awake hand surgery. Hand Clin 2019;35(1):1-6. DOI: 10.1016/j.hcl.2018.08.002.

6. Codding JL, Bhat SB, llyas AM. An economic analysis of MAC versus WALANT: a trigger finger release surgery case study. Hand 2017;12(4):348-351. DOI: 10.1177/1558944716669693.

7. Wang K, Wang Q, Chen Z. A case of hand surgeon infection of COVID19 due to exposure to treating a suspect patient. Chinese J Hand Surg 2020;36. DOI: 10.3760/cma.j.cn311653-20200304-00096.

8. Ducournau F, Arianni M, Awwad S, et al. COVID-19: initial experience of an international group of hand surgeons. Hand Surg Rehabilitat 2020;39(3):159-166. DOI: 10.1016/j.hansur.2020.04.001.

9. Picardo $\mathrm{NE}$, Walker $\mathrm{H}$, Vanat $\mathrm{Q}$, et al. Service reconfiguration in the department of hand surgery during the UK COVID-19 lockdown: Birmingham experience. Postgrad Med J 2021. DOI: 10.1136/ postgradmedj-2020-139280postgradmedj-2020-139280.

10. Venkatesan A, lyengar K, Loh WYC. Strategies in reconfiguration of hand injuries management during COVID-19 pandemic. J Clin Orthop Trauma 2020;11(4):697-699. DOI: 10.1016/j.jcot.2020. 05.020 . 